\title{
Somatotype, the risk of hydroxychloroquine retinopathy, and safe daily dosing guidelines
}

This article was published in the following Dove Press journal: Clinical Ophthalmology

\section{David J Browning \\ Chong Lee}

Department of Ophthalmology, Charlotte Eye, Ear, Nose and Throat Associates, PA, Charlotte, NC, USA
Correspondence: David J Browning Department of Ophthalmology, Charlotte Eye, Ear, Nose and Throat Associates, PA, 6035 Fairview Road, Charlotte, NC 28210, USA

$\mathrm{Tel}+\mathrm{I} 7042953180$

Fax +I 7042953186

Email dbrowning@ceenta.com
Purpose: The aim of this study was to determine whether somatotype influences the risk of hydroxychloroquine (HC) retinopathy (HCR) and whether dosing by real body weight (RBW), ideal body weight (IBW), or the lesser of these better predicts the risk of HCR.

Patients and methods: A total of 565 patients taking HC for whom height and weight were recorded and a sensitive ancillary testing modality was used including 10-2 visual fields, spectral domain optical coherence tomography, fundus autofluorescence imaging, and multifocal electroretinography were enrolled. Body mass index (BMI) was compared for patients without and with HCR. Logistic regression models of age, cumulative dose, and daily dosing based on RBW, IBW, or lesser of these were compared. Area under the curve (AUC) of receiver operating characteristic plots was used to assess the diagnostic accuracy of RBW, IBW, and lesser of these guidelines for safe dosing. Probability plots for the risk of retinopathy versus BMI were compared for the different recommended guidelines on safe dosing.

Results: A total of 41 patients had HCR. The median BMI was 27.6 (interquartile range [IQR] $24.3,32.6)$ and 24.0 (IQR 21.0, 31.6) for patients without and with HCR $(P=0.0102)$, respectively. AUC for univariate receiver operating characteristic plots of retinopathy versus dosing by RBW, IBW, and lesser of these was $0.71,0.72$, and 0.76 , respectively. AUC for multivariate receiver operating characteristic plots of retinopathy versus models incorporating gender, age, cumulative dose, and BMI and differing by including dosing by RBW, IBW, and lesser of these was $0.82,0.82$, and 0.83 , respectively. For all of the multivariate logistic models, the risk of retinopathy was higher for lower BMIs.

Conclusion: Short, asthenic women are at higher risk for HCR. The 2011 American Academy of Ophthalmology (AAO) guidelines are safer for short, obese women. The 2016 AAO guidelines are safer for short, asthenic patients. Choosing daily dosing based on the lesser of the RBW and IBW guidelines is safer for all patients.

Keywords: hydroxychloroquine, hydroxychloroquine retinopathy, dosing guidelines, American Academy of Ophthalmology guidelines, somatotype

\section{Introduction}

Of all the patient risk factors for hydroxychloroquine (HC) retinopathy (HCR), the only one that is modifiable is daily dosing. Cumulative dose, duration of therapy, renal and liver insufficiency, gender, race, age, pre-existing macular status, and concomitant use of tamoxifen are fixed. Therefore, an emphasis on guidelines for safe dosing is appropriate, since overdosing is common. ${ }^{1-4}$ The dependence of safe dosing on real body weight (RBW) versus ideal body weight (IBW), and somatotype, has been a topic of discussion since HCR was first described, but the prevailing viewpoint has fluctuated. Weight is especially important for $\mathrm{HC}$ dosing because of the known sequestration of HC away from fatty tissues. ${ }^{11,13-15}$ 
The literature before 2013 suggested that short obese women are at higher risk. ${ }^{5}$ In 2013, we reported data indicating that this generalization was untrue and that instead, short, asthenic women were at higher risk. ${ }^{2}$ Other studies later confirmed these observations but went further and proposed a new, purportedly safer, method for dosing HC. ${ }^{6,7}$ American Academy of Ophthalmology (AAO) guidelines had formerly emphasized dosing based on IBW, and our study suggested that the guidelines should be based on the lesser of RBW and IBW. ${ }^{2}$ The 2016 version of the AAO guidelines suggested that dosing should be based on RBW using a new dosing conversion factor of $5 \mathrm{mg} / \mathrm{kg} / \mathrm{d}$ rather than the previous conversion factor of $6.5 \mathrm{mg} / \mathrm{kg} \mathrm{IBW} / \mathrm{d} .^{7}$

The report by Melles and Marmor ${ }^{6}$ led to changes in the AAO guidelines, but their results have not been replicated, and they allow higher doses of $\mathrm{HC}$ for short, obese patients than previous guidelines. Moreover, they compared dosing by RBW and IBW, but not by the lesser of RBW and IBW as we had recommended. They also analyzed the risk of retinopathy in their data set using a univariate methodology rather than a multivariate methodology, which failed to account for the effects of covariates of potential importance. In this work, using an independent data set, we examine the replicability of the findings of Melles and Marmor, expand the analysis to include dosing by the lesser of RBW and IBW, and use multivariate analysis rather than univariate analysis to assess the risk of retinopathy.

\section{Patients and methods}

This was a retrospective study of 865 patients taking HC for autoimmune diseases who were referred for retinopathy screening drawn from the private group practice of 34 ophthalmologists and four optometrists. Patients were excluded if there was no information on height or weight in the chart. All patients had one or more of the following ancillary tests done: 10-2 visual field (10-2 VF) testing, spectral domain optical coherence tomography (SD-OCT), multifocal electroretinography (mfERG), and fundus autofluorescence (FAF) imaging. Patients were diagnosed as having retinopathy based on the totality of the ancillary test evidence such that the drug was discontinued. All ancillary testing was reviewed by DJB. If this independent review disagreed with the clinical diagnosis, the case was considered as showing no retinopathy even if $\mathrm{HC}$ had been discontinued.

Body mass index (BMI) was calculated as weight in kilogram/(height in meters). ${ }^{2}$ All standard automated perimetry was carried out with the Humphrey Visual Field Analyzer using the 10-2 program with either a white test object or a red test object. SD-OCT images were taken using either a
Heidelberg SPECTRALIS instrument or a Zeiss CIRRUS instrument. mfERG was carried out using a Espion instrument (Diagnosys, Lowell, MA, USA). FAF images were taken using a Retinal Analyzer (Heidelberg, Franklin, MA, USA). For this retrospective study of anonymized medical records and clinical images, waiver of informed consent and waiver of Health Insurance Portability and Accountability Act Authorization were approved by the Presbyterian Hospital Institutional Review Board (IRB; \# 12053). The authors practice at Charlotte Eye, Ear, Nose and Throat Associates. The IRB is associated with Presbyterian Hospital, a public hospital serving Charlotte, NC, USA, and the surrounding region.

Statistical analyses were performed using JMP software (Version 4.0; SAS Institute Inc., Cary, NC, USA) and MedCalc Statistical Software Version 15.11.1 (MedCalc Software bvba, Ostend, Belgium). Fisher's exact test was used for hypothesis testing of differences in proportions. The Kruskal-Wallis test was used to test differences between nonnormally distributed continuous measurements (eg, weight, height, and BMI). Receiver operating characteristic curves were constructed, and the area under the curve (AUC) measurements were assessed and compared between different predictor models using the DeLong method. Multivariable logistic regression was used to develop a model predicting HCR from risk factors. The multivariable model included significant predictor variables for HCR in univariate logistic regression. A backward-stepwise approach was used to remove nonsignificant terms if $P>0.10$. An $\alpha$ of 0.05 was chosen as the threshold for statistical significance.

\section{Results}

Of the 865 patients whose charts were reviewed, 298 patients lacked height or weight, leaving 567 patients for analysis, of whom two patients lacked any of 10-2 VF, SD-OCT, or mfERG. The analyzed data set included 565 patients. The relative frequency of specific forms of ancillary testing done was assessed; 10-2 VF, SD-OCT, mfERG, 10-2 VF with SD-OCT, and 10-2 VF with mfERG were carried out in 562 (99.5\%), 358 (63.3\%), 240 (42.5\%), 355 (62.8\%), and 240 (42.5\%) patients, respectively. Daily dose and duration of therapy were missing from the charts of 47 and 20 patients, respectively.

Ten patients were Asian, 115 patients were AfricanAmerican, and 418 patients were Caucasian. Other demographic characteristics of these patients are listed in Table 1. Women were significantly more likely to have HCR than men. The weight of patients with retinopathy was significantly less than that of patients without retinopathy. Patients with retinopathy were significantly more likely to take a daily 
Table I Patient demographics

\begin{tabular}{|c|c|c|c|c|}
\hline Characteristic & Entire group & No retinopathy & Retinopathy & $P$ \\
\hline Female & $513(90.8)$ & $472(90.1)$ & $4 I(100)$ & $0.0246^{*}$ \\
\hline Renal disease & $17(3.0)$ & $15(2.9)$ & $2(4.9)$ & $0.3535^{*}$ \\
\hline Liver disease & $8(1.4)$ & $7(1.3)$ & I (2.4) & $0.4536 *$ \\
\hline Both renal and liver disease & $2(0.4)$ & $2(0.4)$ & $0(0)$ & $1.0000 *$ \\
\hline Taking tamoxifen & $3(0.5)$ & $3(0.6)$ & $0(0)$ & $1.0000^{*}$ \\
\hline Weight (lbs), median (IQR) & $160(135,189)$ & $161(135,190)$ & $135(120,177)$ & $0.0026 * *$ \\
\hline Height (in), median (IQR) & $64(62,66)$ & $64(62,66)$ & $63(62,66)$ & $0.1586 * *$ \\
\hline IBW (lbs), median (IQR) & $140(|3|, \mid 48)$ & $140(131, \mid 48)$ & $135(13|| 48)$, & $0.1586 * *$ \\
\hline \multicolumn{5}{|l|}{ Daily dose category (mg) } \\
\hline$\leq 200$ & $172(33.1)$ & $169(35.0)$ & $3(8.6)$ & $0.0002 * * *$ \\
\hline $200<X<400$ & $50(9.6)$ & $49(10.1)$ & I (2.9) & \\
\hline 400 & $297(57.2)$ & $266(55.0)$ & $31(88.6)$ & \\
\hline Duration of therapy (years), median (IQR) & $6.2(2.7,11.9)$ & $5.9(2.5,11.2)$ & $12.7(6.9,17.2)$ & $<0.000 I^{* *}$ \\
\hline Cumulative dose (g), median (IQR) & $694(283,1,424)$ & $670(266,1,306)$ & $1,919(999,2,613)$ & $<0.000 I^{* *}$ \\
\hline $\begin{array}{l}\text { Toxic dosing using } 6.5 \mathrm{mg} / \mathrm{kg} \text { of IBW/d } \\
\text { threshold, NHLBI algorithm }\end{array}$ & $113(21.8)$ & $95(19.7)$ & $18(51.4)$ & $<0.000 I^{*}$ \\
\hline $\begin{array}{l}\text { Toxic dosing using } 6.5 \mathrm{mg} / \mathrm{kg} \text { of IBW/d } \\
\text { threshold, Wolfe algorithm }\end{array}$ & $225(43.3)$ & $198(40.9)$ & $27(77.1)$ & $<0.0001 *$ \\
\hline Toxic dosing using $5 \mathrm{mg} / \mathrm{kg}$ of RBW/d threshold ${ }^{\mathrm{a}}$ & $200(38.6)$ & $178(36.9)$ & $22(62.9)$ & $0.0035^{*}$ \\
\hline $\mathrm{BMI}\left(\mathrm{kg} / \mathrm{m}^{2}\right)$ & $27.4(24.0,32.5)$ & $27.6(24.4,32.6)$ & $24.0(21.0,31.6)$ & $0.0103^{*}$ \\
\hline
\end{tabular}

Notes: Data are presented as n (\%) unless otherwise specified. The number of patients (denominator) for the column "Entire group" was 565 , for the column "No retinopathy" was 524, for the column "Retinopathy" was 4 I. P, P-value from the statistical hypothesis test comparing the values in the columns "No retinopathy" and "Retinopathy". *Fisher's exact test. **Kruskal-Wallis test. ****Chi-square (likelihood ratio) test. alnformation on daily dosing was missing for 47 of the 565 patients, of whom $4 \mathrm{I}$ and six patients were in the groups without and with retinopathy, respectively.

Abbreviations: BMI, body mass index; IQR, interquartile range; IBW, ideal body weight; NHLBI, National Heart Lung and Blood Institute; RBW, real body weight.

dose of $400 \mathrm{mg} / \mathrm{d}$ than patients without retinopathy. Patients with retinopathy were more often overdosed based on IBW guidelines regardless of IBW algorithm used and were also more often overdosed based on RBW guidelines. ${ }^{5,78}$ BMI was less in patients with retinopathy compared to patients without retinopathy. The proportions of patients with renal disease and liver disease were not significantly different between those with and without retinopathy. The proportions of patients who were Asian, African-American, and Caucasian did not differ between those with and without retinopathy.

In a univariate logistic analysis of retinopathy versus patient characteristics, the following factors were statistically significant, followed by their $P$-values: age ( 0.0033$)$, gender (0.0246), daily dose category (0.0002), duration $(<0.0001)$, cumulative dose $(<0.0001)$, RBW (0.0061), lesser of RBW and IBW $(<0.0001)$, daily dose based on RBW $(<0.0001)$, daily dose based on IBW $(<0.0001)$, daily dose based on the lesser of RBW and IBW $(<0.0001)$, and BMI (0.0192).

The following characteristics were not significant: IBW (0.1263), renal disease (0.3535), liver disease (0.4548), race (Asian [0.5322], African-American [0.1580], and Caucasian [0.2662]), and tamoxifen use (1.000).

Receiver operating curves were used to quantitate the diagnostic accuracy of the IBW, RBW, and lesser of IBW and RBW methods of daily dosing in predicting the risk of retinopathy. In these models, the greater the AUC, the greater the accuracy in predicting retinopathy. The AUCs for the three univariate models in which one of the three methods of daily dosing was used to predict retinopathy were 0.71 , 0.72 , and 0.76 for IBW, RBW, and the lesser of IBW and $\mathrm{RBW}$, respectively (Figure 1). The base for the multivariable

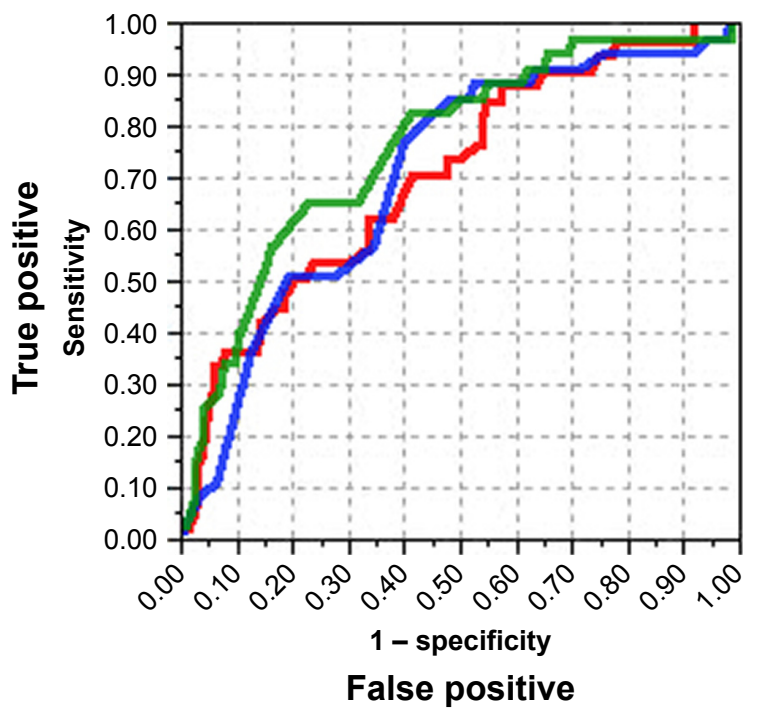

Figure I Receiver operating characteristic curves for hydroxychloroquine retinopathy predicted by dosing guidelines as a univariate analysis.

Note: The red curve is the RBW method, the blue curve is the IBW method, and the green curve is the lesser of IBW and RBW methods.

Abbreviations: IBW, ideal body weight; RBW, real body weight. 
model included the variables gender, age, cumulative dose, and BMI. This base model was then varied by adding one of the following three methods of daily dosing: daily dosing based on IBW, daily dosing based on RBW, and daily dosing based on the lesser of IBW and RBW. The AUCs for the three multivariate models using gender, age, cumulative dose, and BMI as common predictive variables, but differing in their use of daily dosing according to the three options of IBW, RBW, and the lesser of IBW and RBW, were $0.82,0.82$, and 0.83 , respectively (Figure 2).

For all of the multivariate logistic models of risk of retinopathy, the risk was higher for lower BMIs (Figure 3); that is, the risk of retinopathy depends on somatotype with short, asthenic patients at higher risk. When risk was plotted for the subgroups taking greater than or equal to the published safe thresholds for the IBW and RBW methods, the risk of retinopathy was higher for lower BMIs for both methods in the subgroups above safe thresholds and invariant in the groups below safe thresholds (Figure 4).

To assess the proportion of patients that would be more safely dosed by the IBW and RBW methods, we plotted height versus RBW (Figure 5). A line can be drawn for which the RBW and IBW methods yield the same threshold for safe dosing. ${ }^{9}$ The line depends on the IBW algorithm chosen. Figure 5 shows the line corresponding to the algorithm used

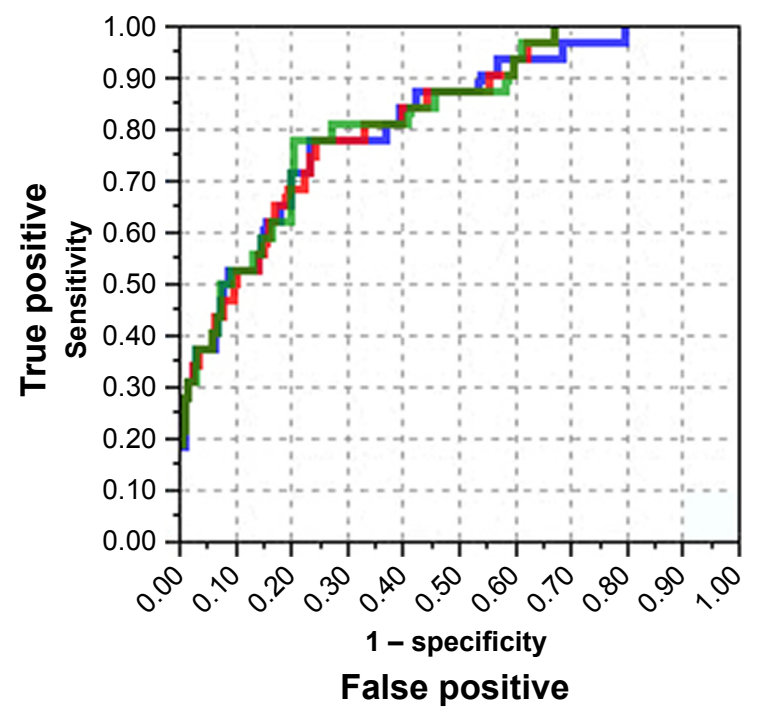

Figure 2 Receiver operating characteristic curves for hydroxychloroquine retinopathy predicted by dosing guidelines as a multivariate analysis.

Notes: Logistic regression models were fit including gender, age, cumulative dose, and $\mathrm{BMI}$ as common predictive variables. The red curve corresponds to the model adding guidelines based on RBW dosing, the blue curve corresponds to the model adding guidelines based on IBW dosing, and the green curve corresponds to the model adding guidelines based on the lesser of IBW and RBW dosing.

Abbreviations: BMI, body mass index; IBW, ideal body weight; RBW, real body weight. by Melles and Marmor ${ }^{6}$ (a modified Devine algorithm in which IBW for all patients of height $\leq 60$ in is arbitrarily set to 100 pounds). For patients whose heights and weights place them above the line, the RBW method yields safer (ie, lower) dosing guidance. For patients below the line, the IBW method yields the safer dosing guidance. For the algorithm chosen by Melles and Marmor, $48.9 \%$ of patients would receive safer dosing guidance when the IBW method is used.

The average weight of people in the USA has been increasing monotonically for the past 50 years. Figure 6 shows the average weight of a woman in the age decile 50-59 years from the National Health and Nutrition Examination Survey. ${ }^{10}$ This decile characterizes the weight of the average person taking $\mathrm{HC}$, a woman in her 50s. Over this interval, the average weight has increased 27.5 pounds, an $18.8 \%$ increase. This translates into an increase in safe dose of $\mathrm{HC}$ by the RBW method of $63 \mathrm{mg} / \mathrm{d}$. IBW depends on height, and by this method, no increase in safe dose over the past 50 years has occurred. The effect of increasing population weight over time has been to drive patients to the right in Figure 6, placing a monotonically higher proportion in the zone for which the IBW method provides safer dosing guidance.

\section{Discussion}

Allusions in the literature that short, obese women are at particular risk for $\mathrm{HC}$ retinopathy were common through the AAO guidelines of 2011.,11 In 2013, we reported that this risk profile was erroneous. ${ }^{2}$ In fact, short, asthenic women were the ones at particular risk and daily dosing should be based on the lesser of RBW and IBW. ${ }^{2}$ Other studies later confirmed the particular risk of the short, asthenic woman but proposed that daily dosing be based on RBW using a conversion factor of $5 \mathrm{mg} / \mathrm{kg} / \mathrm{d} .{ }^{6}$ This proposal takes care of the problem of short, asthenic women but puts short, obese women at risk, as published cases document and as inspection of Melles and Marmor's own data reveals (Figure 7). ${ }^{6,12}$ Using the lesser of the RBW and IBW guidelines takes care of the short, asthenic patient and also provides safer dosing for the short, obese woman. A convenient smartphone application (DoseChecker) has been designed to provide safer dosing using this approach. ${ }^{9}$

Melles and Marmor ${ }^{6}$ have reported that the univariate AUCs for the receiver operating characteristic curves of RBW and IBW in predicting retinopathy were 0.78 and 0.75 , respectively. In the independent data set of this study, we found the corresponding values of 0.72 and 0.71 , 
A

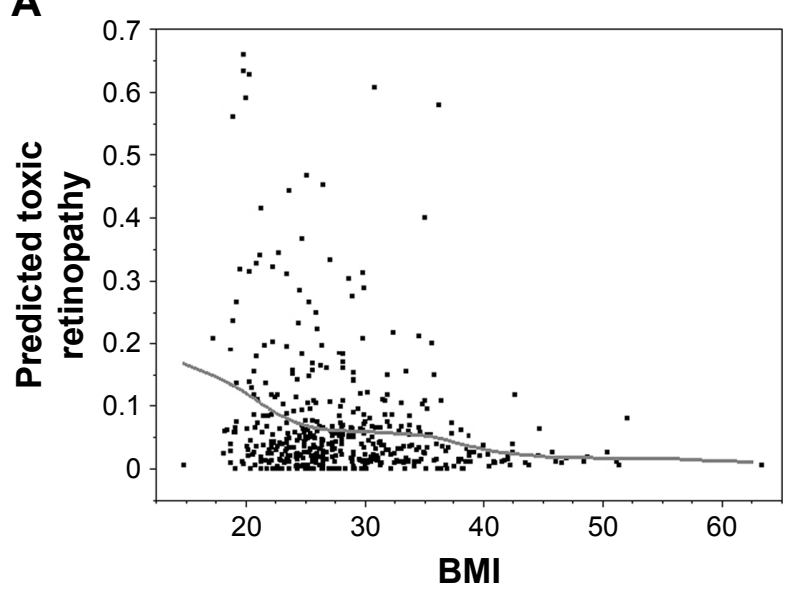

B

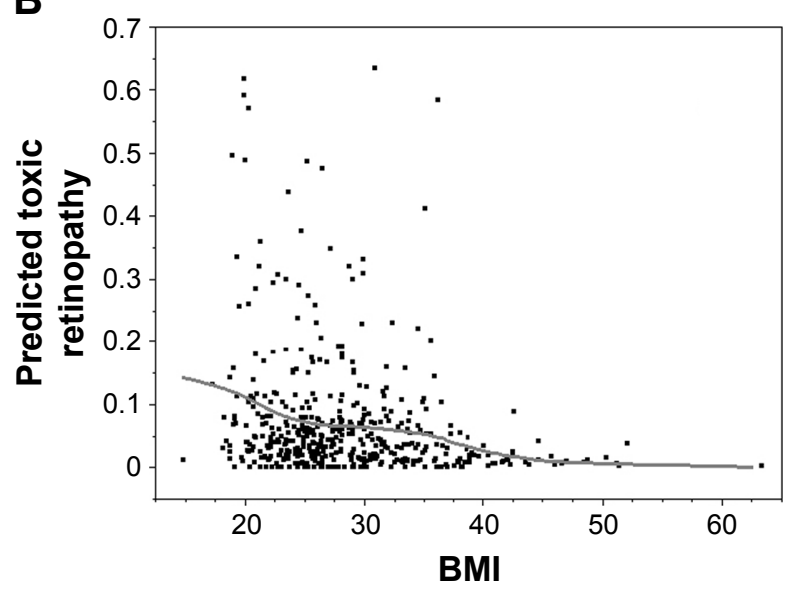

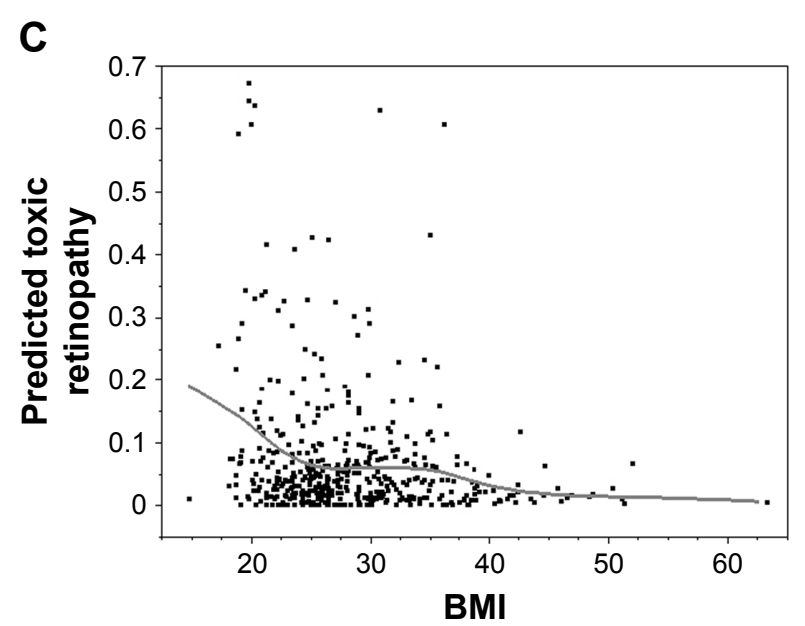

Figure 3 Predicted risk of hydroxychloroquine retinopathy versus BMI from multivariate logistic models with a common base model including gender, age, cumulative dose, and BMI, and differing according to whether the model included dosing guidelines based on RBW (A), IBW (B), or the lesser of RBW and IBW (C). Notes: Each point refers to a single patient. The black curve is a spline fit of predicted risk to the data points with $\lambda$ (a smoothing parameter) $=I, 000$. Abbreviations: BMI, body mass index; IBW, ideal body weight; RBW, real body weight.
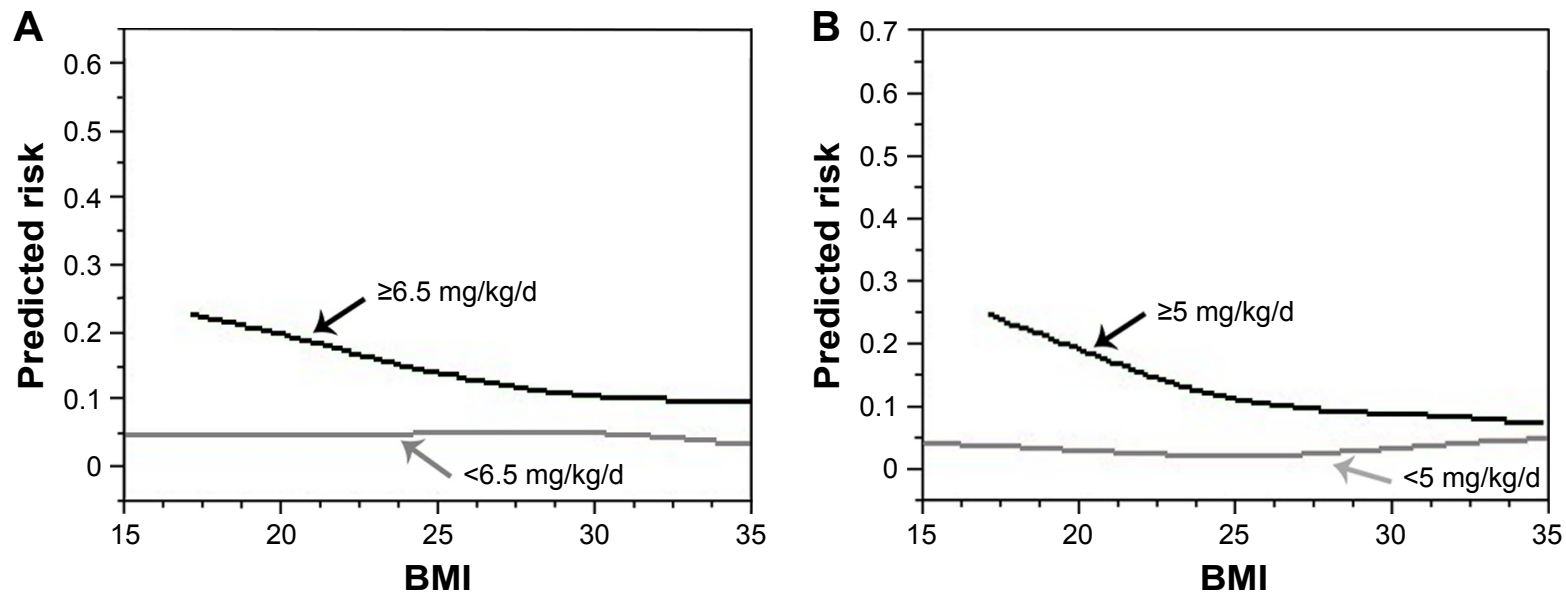

Figure 4 Predicted risk of hydroxychloroquine retinopathy versus BMI from multivariate logistic regression models as described in Figure 3.

Notes: (A) The predicted risk for the subgroups of patients taking $\geq 6.5 \mathrm{mg} / \mathrm{kg} / \mathrm{d}$ (black line) and $<6.5 \mathrm{mg} / \mathrm{kg} / \mathrm{d}$ (gray line) of ideal body weight, respectively. (B) The predicted risk for the subgroups of patients taking $\geq 5.0 \mathrm{mg} / \mathrm{kg} / \mathrm{d}$ (black line) and $<5.0 \mathrm{mg} / \mathrm{kg} / \mathrm{d}$ (gray line) of real body weight, respectively. The black and gray curves are spline fits of predicted risk to the data points with $\lambda$ (a smoothing parameter) $=I, 000$. The data points are not shown.

Abbreviation: BMI, body mass index. 


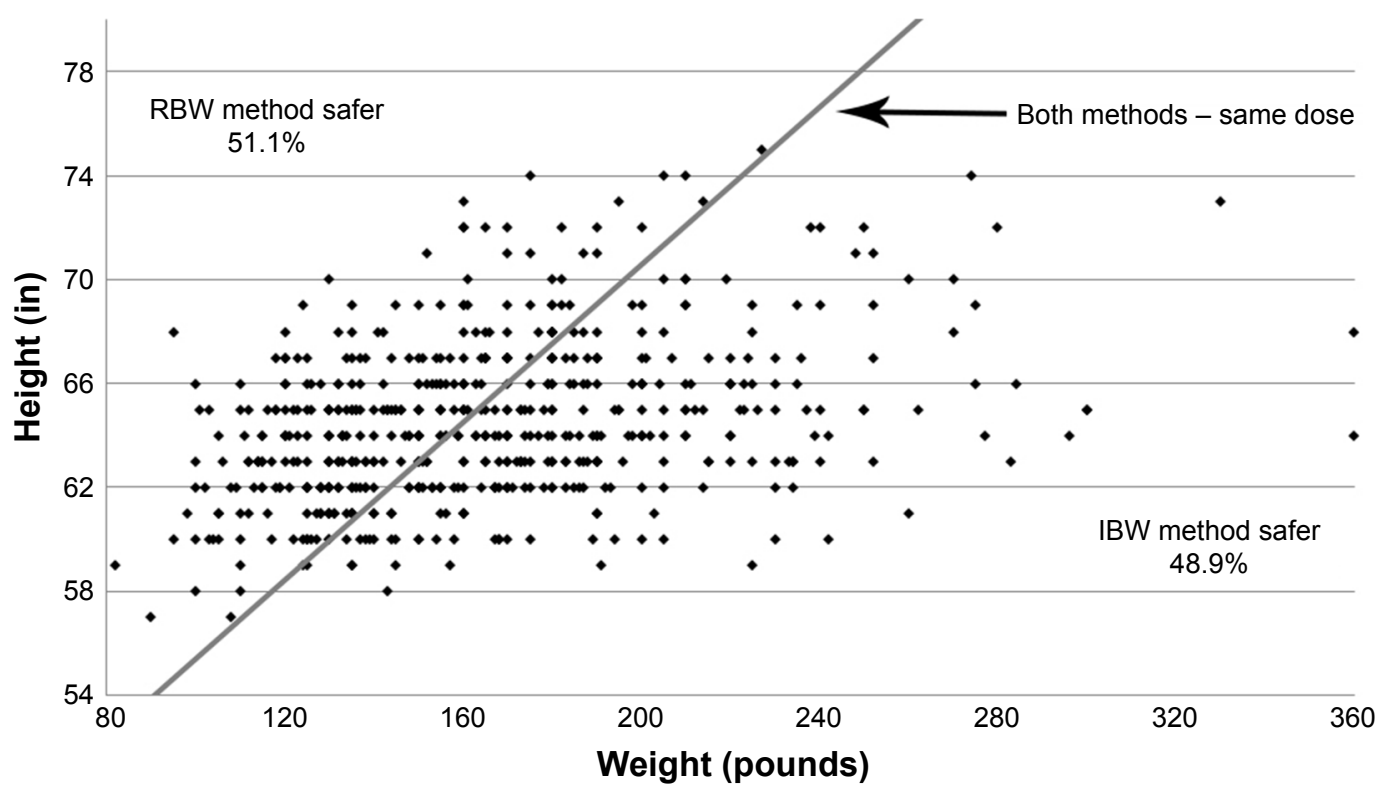

Figure 5 Allocation of patients into zones of safer dosing by the IBW or RBW methods using the IBW algorithm published by Melles and Marmor. ${ }^{6}$ Notes: Each point refers to a patient. For patients who fall on the line, both methods yield the same recommended maximal safe dose. For patients who fall above the line, the RBW method yields a safer dose. For patients who fall below the line, the IBW method yields a safer dose.

Abbreviations: IBW, ideal body weight; RBW, real body weight.

respectively. In these univariate analyses, the differences in the AUC of 0.03 and 0.01 have no clinical importance, although the 0.03 difference achieved statistical significance in the Melles and Marmor study because of a sufficiently large sample size $(\mathrm{N}=2,361)$. They did not examine their data set for the predictive power of the lesser of RBW and IBW nor did they use multivariate logistic regression to include other risk factors besides daily dosing guidelines. We included for comparison the lesser of RBW and IBW as a predictive variable for toxicity and performed multivariate analyses including other significant risk factors.

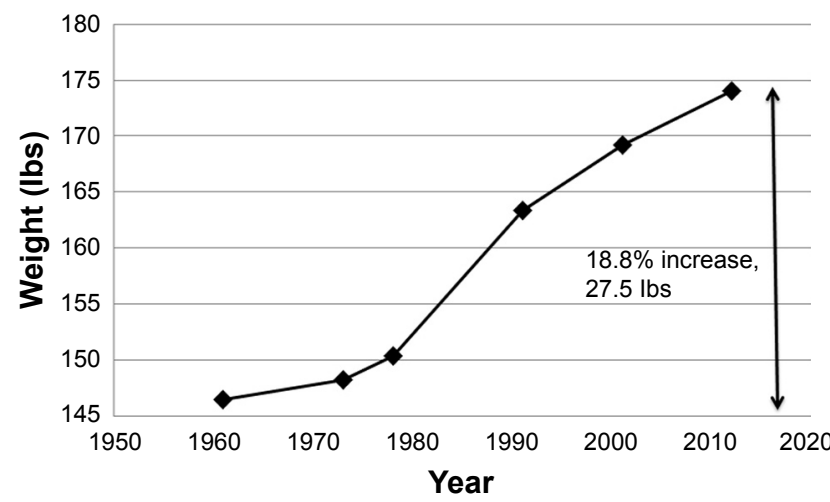

Figure 6 Graph of the average weight for a woman in the age decile 50-59 years in the USA over the time span 1960-2012 from the NHANES.

Notes: The average patient taking hydroxychloroquine falls into this gender and age decile. The average weight of such a person has increased 27.5 pounds (18.8\%). Abbreviation: NHANES, National Health and Nutrition Examination Survey.
In contrast to the conclusion of Melles and Marmor, we found no evidence that the RBW method predicted retinopathy better than the IBW or lesser of RBW and IBW methods based on multivariate analysis. Thus, the evidence upon which AAO safe dosing guidelines changed in 2016 has not been replicated and the wisdom of the change is questionable, given that population median RBWs continue to rise over time implying an increasing safe ceiling for $\mathrm{HC}$

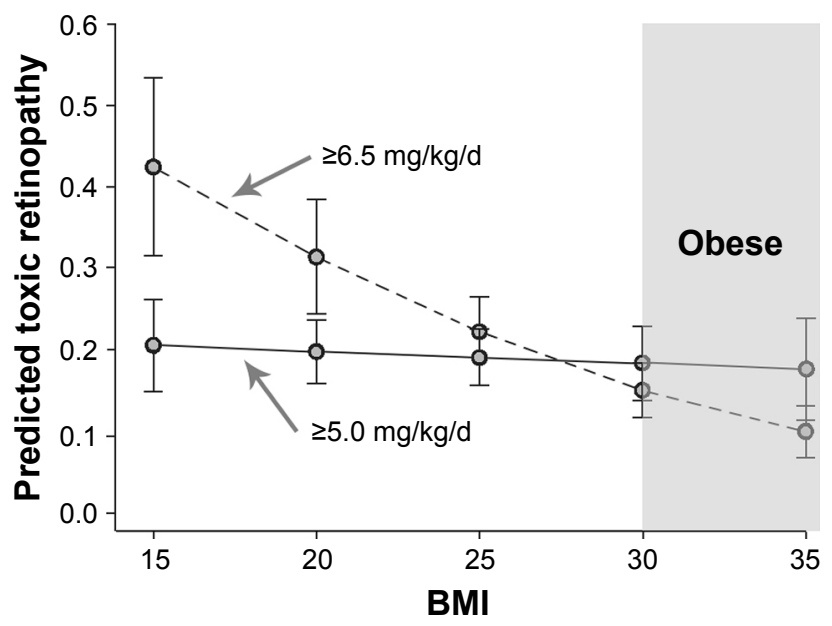

Figure 7 Risk versus body habitus based on ideal body weight and real body weight.

Notes: The data show that for obese patients (those with BMI $>30$ ), the risk of retinopathy was lower with the ideal body weight method of daily dosing compared to the real body weight method. Data from Melles and Marmor. ${ }^{6}$

Abbreviation: BMI, body mass index. 
dosing (Figure 7), an illogical implication at odds with known sequestration of $\mathrm{HC}$ away from fatty tissues. ${ }^{11,13-15}$

The analysis of our data set confirmed the analysis of the Melles and Marmor ${ }^{6}$ data set showing that predicted risk of retinopathy using the IBW method of daily dosing and a $6.5 \mathrm{mg} / \mathrm{kg}$ threshold for safe dosing increases for lower BMIs. However, we were not able to confirm their analysis using the RBW method of daily dosing with a threshold dose of $5 \mathrm{mg} / \mathrm{kg} / \mathrm{d}$ for safe dosing. Melles and Marmor ${ }^{6}$ found that for patients whose daily doses were $\geq 5 \mathrm{mg} / \mathrm{kg}$ $\mathrm{RBW} / \mathrm{d}$, the predicted risk of retinopathy was invariant with BMI. In contrast, our data set showed a higher risk of retinopathy with lower BMIs similar to the relationship found using the IBW method of daily dosing. To resolve the different findings, a prospective study using a large sample from multiple centers would be desirable. The recent expansion of the remit of the Diabetes Retinopathy Clinical Research network to study other retinal diseases provides an optimal platform to conduct a study that could answer this question.

Although the daily dose adjusted by some version of the patient's mass is the only modifiable variable, there are unknown variables yet to be identified. This is highlighted by the group of patients who had retinopathy but never had toxic dosing. The proportion of these was 17 (48.6\%), 13 (37.1\%), and $12(34.3 \%)$ for daily dosing by IBW (National Heart Lung and Blood Institute algorithm, $6.5 \mathrm{mg} / \mathrm{kg} / \mathrm{d}$ conversion factor), daily dosing by RBW ( $5 \mathrm{mg} / \mathrm{kg} / \mathrm{d}$ conversion factor), and daily dosing by lesser of IBW and RBW, respectively.

Limitations of our study include a patient setting that may not be generalizable, retrospective data collection, use of a single grader for determining retinopathy, and dependence on patient report regarding medication use, liver function, and renal function. These limitations are common to previous studies, and there are no prospective, level I studies of HCR that do not suffer from similar flaws.

\section{Conclusion}

Short, asthenic women comprise a somatotype at increased risk for HCR. Basing $\mathrm{HC}$ dosing on the lesser of the RBW and IBW methods is safer for both short, asthenic and short, obese patients. Using the RBW method for all patients as recommended by the AAO 2016 guidelines places short, obese patients at unnecessarily increased risk for retinopathy.
The DoseChecker smartphone application is a simple way for the clinician to incorporate these principles into practice. ${ }^{9}$

\section{Acknowledgment}

There is no financial relationship of the two entities.

\section{Disclosure}

The authors report no conflicts of interest in this work.

\section{References}

1. Browning DJ. The prevalence of hydroxychloroquine retinopathy and toxic dosing, and the role of the ophthalmologist in reducing both. Am J Ophthalmol. 2016;166:ix-xi.

2. Browning DJ. Impact of the revised American academy of ophthalmology guidelines regarding hydroxychloroquine screening on actual practice. Am J Ophthalmol. 2013;155(3):418.e1-428.e1.

3. Braslow RA, Shiloach M, Macsai MS. Adherence to hydroxychloroquine dosing guidelines by rheumatologists. An electronic medical recordbased study in an integrated health care system. Ophthalmology. 2017; 124(5):604-608.

4. Au A, Parikh V, Modi YS, Ehlers JP, Schachat AP, Singh RP. Hydroxychloroquine screening practice patterns within a large multispecialty ophthalmic practice. Am J Ophthalmol. 2015;160(3):561-568.

5. Marmor MF, Kellner U, Lai TY, Lyons JS, Mieler WF; American Academy of Ophthalmology. Revised recommendations on screening for chloroquine and hydroxychloroquine retinopathy. Ophthalmology. 2011;118(2):415-422.

6. Melles RB, Marmor MF. The risk of toxic retinopathy in patients on long-term hydroxychloroquine therapy. JAMA Ophthalmol. 2014; 132(12):1453-1460.

7. Marmor MF, Kellner U, Lai TY, Melles RB, Mieler WF; American Academy of Ophthalmology. Recommendations on screening for chloroquine and hydroxychloroquine retinopathy (2016 revision) Ophthalmology. 2016;123(6):1386-1394.

8. Browning DJ, Lee C, Rotberg D. The impact of different algorithms for ideal body weight on screening for hydroxychloroquine retinopathy in women. Clin Ophthalmol. 2014;8:1401-1407.

9. Perlman EM, Greenberg PB, Browning DJ, Friday RP, Miller JW Solving the hydroxychloroquine dosing dilemma with a smartphone app. JAMA Ophthalmol. 2017;136(2):218-219.

10. Ogden CL, Fryar CD, Carroll MD, Flegal KM. Mean body weight, height, and body mass index, United States 1960-2002. Adv Data. 2004;347:1-17.

11. Browning DJ. Hydroxychloroquine and Chloroquine Retinopathy. 1 st ed. New York: Springer; 2014:170.

12. Browning DJ. Avoiding the traps of hydroxychloroquine use. Rev Ophthalmol. 2016. Available from: https://www.reviewofophthalmology. com/article/avoiding-the-traps-of-hydroxychloroquine-use. Accessed January 23, 2018.

13. Mcchesney EW. Animal toxicity and pharmacokinetics of hydroxychloroquine sulfate. Am J Med. 1983;75(1A):11-18.

14. Mcchesney EW, Shekosky JM, Hernandez PH. Metabolism of chloroquine-3-14C in the rhesus monkey. Biochem Pharmacol. 1967;16(2): 2444-2447.

15. Mcchesney EW, Conway WD, Banks WF Jr, Rogers JE, Shekosky JM. Studies of the metabolism of some compounds of the 4-amino-7chloroquinoline series. J Pharmacol Exp Ther. 1966;151(3):482-493. 


\section{Publish your work in this journal}

Clinical Ophthalmology is an international, peer-reviewed journal covering all subspecialties within ophthalmology. Key topics include: Optometry; Visual science; Pharmacology and drug therapy in eye diseases; Basic Sciences; Primary and Secondary eye care; Patient Safety and Quality of Care Improvements. This journal is indexed on

Submit your manuscript here: http://www.dovepress.com/clinical-ophthalmology-journal
PubMed Central and CAS, and is the official journal of The Society of Clinical Ophthalmology (SCO). The manuscript management system is completely online and includes a very quick and fair peer-review system, which is all easy to use. Visit http://www.dovepress.com/ testimonials.php to read real quotes from published authors. 